\title{
Higher form gauge fields and their nonassociative symmetry algebras
}

\section{Leonardo Castellani}

Dipartimento di Scienze e Innovazione Tecnologica, Università del Piemonte Orientale and INFN Sezione di Torino,

Viale T. Michel 11, 15121 Alessandria, Italy

E-mail: leonardo.castellani@mfn.unipmn.it

ABSTRACT: We show that geometric theories with $p$-form gauge fields have a nonassociative symmetry structure, extending an underlying Lie algebra. This nonassociativity is controlled by the same Chevalley-Eilenberg cohomology that classifies free differential algebras, $p$-form generalizations of Cartan-Maurer equations. A possible relation with flux backgrounds of closed string theory is pointed out.

KEYwORDs: Differential and Algebraic Geometry, Flux compactifications, Supergravity Models

ARXiv EPRINT: 1310.7185 
Free differential algebras (FDA's) [1-4] (for reviews see for ex. [5, 6]) provide a convenient algebraic setting for field theories with antisymmetric tensors. They generalize the Cartan-Maurer equations satisfied by the 1-form vielbeins on a group manifold $G$ by including $p$-form fields, and have been extensively used in the construction of (super)gravity theories. Given a FDA, there is a well-defined procedure to obtain a lagrangian for a field theory containing the $p$-forms of the FDA as fundamental fields.

Their dual formulation, based on the algebra of Lie derivatives on the "FDA manifold", has been studied in refs. [7-9], and uses an extended Lie derivative along these antisymmetric tensors.

In the present paper we show that the resulting algebra of Lie derivatives is nonassociative, and that the nonassociativity is controlled by the same Chevalley-Eilenberg cohomology that classifies FDA's. In fact, our interest in studying the nonassociativity of FDA dual algebras has been prompted by a recent paper [10], where flux backgrounds in closed string theory are described by nonassociative structures in double phase space, controlled again by Chevalley-Eilenberg cohomology (for a very partial list of references see for example [11-14]). Since flux backgrounds involve $p$-forms, it seems that algebraic structures describing $p$-forms tend to exhibit nonassociativity, depending on nontrivial cohomology classes, both for flux backgrounds and in FDA's dual algebras. A clue for a possible relation is given in the example i) (see below), where the nonassociative algebra of the R-flux model in $[10,11]$ is identified with a particular FDA dual algebra.

Free differential algebras. Consider the case of ordinary Cartan-Maurer one-forms $\sigma^{A}$ supplemented by a single $p$-form $B^{i}$ in a representation $D^{i}{ }_{j}$ of $G$ :

$$
\begin{aligned}
& d \sigma^{A}+\frac{1}{2} C^{A}{ }_{B C} \sigma^{B} \sigma^{C}=0 \\
& d B^{i}+C^{i}{ }_{A j} \sigma^{A} B^{j}+\frac{1}{(p+1) !} C^{i}{ }_{A_{1} \ldots A_{p+1}} \sigma^{A_{1}} \ldots \sigma^{A_{p+1}} \\
& \quad \equiv \nabla B^{i}+\frac{1}{(p+1) !} C_{{ }_{1} \ldots A_{p+1}}^{i} \sigma^{A_{1}} \ldots \sigma^{A_{p+1}}=0
\end{aligned}
$$

Products between forms are understood to be exterior products. Taking the exterior derivative of the left-hand sides of (1), (2) and requiring $d^{2}=0$ translates into a set of conditions for the structure constants:

$$
\begin{aligned}
C^{A}{ }_{B[C} C^{B}{ }_{D E]} & =0 \\
C^{i}{ }_{A j} C^{j}{ }_{B k}-C^{i}{ }_{B j} C^{j}{ }_{A k} & =C^{C}{ }_{A B} C^{i}{ }_{C k} \\
2 C^{i}{ }_{\left[A_{1} j\right.} C^{j}{ }_{\left.A_{2} \ldots A_{p+2}\right]}-(p+1) C^{i}{ }_{B\left[A_{1} \ldots A_{p}\right.} C^{B}{ }_{\left.A_{p+1} A_{p+2}\right]} & =0
\end{aligned}
$$

Eqs. (3) are the usual Jacobi identities for the Lie algebra $G$. Eq. (4) implies that $\left(C_{A}\right)^{i}{ }_{j} \equiv$ $C^{i}{ }_{A j}$ is a matrix representation of $G$, while eq. (5) states that $C^{i} \equiv C^{i}{ }_{A_{1} \ldots A_{p+1}} \sigma^{A_{1}} \ldots \sigma^{A_{p+1}}$ is a $(p+1)$-cocycle, i.e. $\nabla C^{i}=0$. Clearly $C^{i}$ differing by covariantly exact pieces $\Phi^{i}$ $\left(\nabla \Phi^{i}=0\right)$ lead to equivalent FDA's via the redefinition $B^{i} \rightarrow B^{i}+\Phi^{i}$. Thus nontrivial FDA extensions of the Lie algebra are classified by nontrivial cohomology classes of the covariant derivative $\nabla$, i.e. by Chevalley cohomology. 
As an example we recall the FDA of $\mathrm{D}=11$ supergravity [1]:

$$
\begin{array}{rlrl}
d \omega^{a b}-\omega^{a c} \omega^{c b}=0 & {\left[=R^{a b}\right]} \\
d V^{a}-\omega^{a b} V^{b}-\frac{i}{2} \bar{\psi} \Gamma^{a} \psi=0 & {\left[=R^{a}\right]} \\
d \psi-\frac{1}{4} \omega^{a b} \Gamma^{a b} \psi & =0 & {[=\rho]} \\
d A-\frac{1}{2} \bar{\psi} \Gamma^{a b} \psi V^{a} V^{b} & =0 & {[=R(A)]}
\end{array}
$$

(all index contractions with the $D=11$ Minkowski metric). The $\mathrm{D}=11$ Fierz identity $\bar{\psi} \Gamma^{a b} \psi \bar{\psi} \Gamma^{a} \psi=0$ ensures the FDA closure $\left(d^{2}=0\right)$. Its Lie algebra part is the $\mathrm{D}=11$ superPoincaré algebra, whose fundamental fields (corresponding to the Lie algebra generators $\left.P_{a}, J_{a b}, Q\right)$ are the vielbein $V^{a}$, the spin connection $\omega^{a b}$ and the gravitino $\psi$. The 3 -form $A$ is in the identity representation of the Lie algebra, and thus no $i$-indices are needed. The structure constants $C^{i}{ }_{A_{1} \ldots A_{p+1}}$ of (2) are in the present case given by $C_{\alpha \beta a b}=-12\left(C \Gamma_{a b}\right)_{\alpha \beta}$ (no upper index $i$ ), while the $C^{i}{ }_{A j}$ vanish. This FDA extension exists thanks to the nontrivial 4-cocycle $\bar{\psi} \Gamma^{a b} \psi V^{a} V^{b}$. The curvatures $R^{a b}, R^{a}, \rho$ and $R(A)$ (i.e. the right-hand sides of the FDA equations, nonvanishing for general field configurations outside the vacuum ${ }^{1}$ ) are respectively the Lorentz curvature, the torsion, the gravitino field strength and the 3 -form field strength. The lagrangian of $d=11$ supergravity can be written as a 11-form, made out of exterior products of fields and curvatures, and is therefore by construction invariant under diffeomorphisms. Infinitesimal diffeomorphisms are generated by Lie derivatives along all the soft group manifold directions, and comprehend all the invariances of the theory. This is essentially the core of the group geometric method developed many years ago $[5,6,15-18]$.

Extended Lie derivatives. Consider the Lie derivatives $\ell_{\varepsilon^{A}} \mathbf{t}_{A}$ along a generic tangent vector $\varepsilon^{A} \mathbf{t}_{A}$, where the $\mathbf{t}_{A}$ are a basis of tangent vectors on $G$ dual to the left invariant one-forms $\sigma^{A}$. By means of the FDA equations and the Cartan formula

$$
\ell_{\varepsilon^{A} \mathbf{t}_{A}}=i_{\varepsilon^{A} \mathbf{t}_{A}} d+d i_{\varepsilon^{A} \mathbf{t}_{A}}
$$

one finds their action on the FDA forms:

$$
\begin{aligned}
\ell_{\varepsilon^{B} \mathbf{t}_{B}} \sigma^{A} & =d\left(i_{\varepsilon^{B} \mathbf{t}_{B}} \sigma^{A}\right)+i_{\varepsilon^{B} \mathbf{t}_{B}} d \sigma^{A} \\
& =d \varepsilon^{A}-C^{A}{ }_{B C} \varepsilon^{B} \sigma^{C} \\
\ell_{\varepsilon^{B} \mathbf{t}_{B}} B^{i} & =d\left(i_{\varepsilon^{B} \mathbf{t}_{B}} B^{i}\right)+i_{\varepsilon^{B} \mathbf{t}_{B}} d B^{i} \\
& =-C_{B j}^{i} \varepsilon^{B} B^{j}-\frac{1}{p !} C_{B A_{1} \ldots A_{p}}^{i} \varepsilon^{B} \sigma^{A_{1}} \ldots \sigma^{A_{p}}
\end{aligned}
$$

Note: the action of the contraction $i_{\mathbf{t}_{B}}$ on the "cotangent FDA basis" $\sigma^{A}, B^{i}$ is given by

$$
i_{\mathbf{t}_{B}} \sigma^{A}=\delta_{B}^{A}, \quad i_{\mathbf{t}_{B}} B^{i}=0
$$

\footnotetext{
${ }^{1}$ In this case we speak of a "soft FDA manifold."
} 
Computing the commutator of two Lie derivatives on $\sigma^{A}$ and $B^{i}$ yields [7-9]:

$$
\begin{aligned}
{\left[\ell_{\varepsilon_{1}^{A} \mathbf{t}_{A}}, \ell_{\varepsilon_{2}^{B} \mathbf{t}_{B}}\right]=} & \ell_{\left[\varepsilon_{1}^{A} \partial_{A} \varepsilon_{2}^{C}-\varepsilon_{2}^{A} \partial_{A} \varepsilon_{1}^{C}+\varepsilon_{1}^{A} \varepsilon_{2}^{B} C_{A B}^{C}\right] \mathbf{t}_{C}} \\
& +\frac{1}{(p-1) !} \ell_{\varepsilon_{1}^{A} \varepsilon_{2}^{B}} C_{A B A_{1} \ldots A_{p-1}}^{i} \sigma^{A_{1} \ldots \sigma^{A}{ }^{A}-1} \mathbf{t}_{i}
\end{aligned}
$$

where the second line involves an extended Lie derivative defined as follows [7]. First introduce a new contraction operator $i_{\varepsilon^{j} \mathbf{t}_{j}}$, acting on a generic form $\omega=$ $\omega_{i_{1} \ldots i_{n} A_{1} \ldots A_{m}} B^{i_{1}} \ldots B^{i_{n}} \sigma^{A_{1}} \ldots \sigma^{A_{m}}$ as

$$
i_{\varepsilon^{j} \mathbf{t}_{j}} \omega=n \varepsilon^{j} \omega_{j i_{2} \ldots i_{n} A_{1} \ldots A_{m}} B^{i_{2}} \ldots B^{i_{n}} \sigma^{A_{1}} \ldots \sigma^{A_{m}}
$$

where $\varepsilon^{j}$ is a (p-1)-form. This operator still maps $p$-forms into $(p-1)$-forms. We can also define the contraction $i_{\mathbf{t}_{j}}$, mapping $n$-forms into $(n-p)$-forms, by setting

$$
i_{\varepsilon^{j} \mathbf{t}_{j}}=\varepsilon^{j} i_{\mathbf{t}_{j}}
$$

In particular

$$
i_{\mathbf{t}_{j}} B^{i}=\delta_{j}^{i}, \quad i_{\mathbf{t}_{j}} \sigma^{A}=0
$$

so that $\mathbf{t}_{j}$ can be seen as the "tangent vector" dual to $B^{j}$.

Note that $i_{\varepsilon} \mathbf{t}_{j}$ vanishes on forms that do not contain at least one factor $B^{i}$. Then the extended Lie derivative is defined by:

$$
\ell_{\varepsilon^{i} \mathbf{t}_{i}} \equiv i_{\varepsilon^{i} \mathbf{t}_{i}} d+d i_{\varepsilon^{i} \mathbf{t}_{i}}
$$

It commutes with $d$, satisfies the Leibniz rule, and can be verified to act on the fundamental fields as

$$
\begin{aligned}
& \ell_{\varepsilon^{j} \mathbf{t}_{j}} \sigma^{A}=0 \\
& \ell_{\varepsilon^{j} \mathbf{t}_{j}} B^{i}=d \varepsilon^{i}+C^{i}{ }_{A j} \sigma^{A} \varepsilon^{j}
\end{aligned}
$$

by using the FDA equations (1), (2). For example if the parameter $(p-1)$-form is given by $\sigma^{A_{1}} \ldots \sigma^{A_{p-1}}$ :

$$
\begin{aligned}
& \ell_{\sigma^{A_{1} \ldots} \sigma^{A_{p-1}} \mathbf{t}_{j}} B^{i}=d\left(\sigma^{A_{1}} \ldots \sigma^{A_{p-1}}\right)+C^{i}{ }_{A j} \sigma^{A} \sigma^{A_{1}} \ldots \sigma^{A_{p-1}}= \\
& \quad=-\left(\frac{p-1}{2} C_{B_{0} B_{1}}^{\left[A_{1}\right.} \delta_{B_{2} \ldots B_{p-1}}^{\left.A_{2} \ldots A_{p-1}\right]} \delta_{j}^{i}-C_{B_{0} j}^{i} \delta_{B_{1} \ldots B_{p-1}}^{A_{1} \ldots A_{p-1}}\right) \sigma^{B_{0}} \ldots \sigma^{B_{p-1}}
\end{aligned}
$$

Using (16) and (17) and the Jacobi identities (3)-(5) it is straightforward to compute the commutators between ordinary and extended Lie derivatives, and between extended Lie derivatives:

$$
\begin{aligned}
{\left[\ell_{\varepsilon^{A} \mathbf{t}_{A}}, \ell_{\varepsilon^{j}} \mathbf{t}_{j}\right] } & =\ell_{\left[\ell_{\varepsilon} A_{\mathbf{t}_{A}} \varepsilon^{k}+C_{B j}^{k} \varepsilon^{B} \varepsilon^{j}\right] \mathbf{t}_{k}} \\
{\left[\ell_{\varepsilon_{1}^{i} \mathbf{t}_{i}}, \ell_{\varepsilon_{2}^{j} \mathbf{t}_{j}}\right] } & =0
\end{aligned}
$$

According to eq. (11) the commutator of two ordinary Lie derivatives contains an extra piece proportional to an extended Lie derivative, that acts nontrivially only on the $p$-form $B^{i}$. Then, if computed on $\sigma^{A}$, the commutator in (11) closes on the usual Lie algebra. Only its action on $B^{i}$ reveals the extra term involving the extended Lie derivative. 
The FDA dual algebra. By taking constant $\varepsilon$ parameters (and nonvanishing only for given directions) the commutations (11), (19) and (20) become:

$$
\begin{aligned}
{\left[\ell_{\mathbf{t}_{A}}, \ell_{\mathbf{t}_{B}}\right] } & =C_{A B}^{C}{ }_{A B} \ell_{\mathbf{t}_{C}}+\frac{1}{(p-1) !} C^{i}{ }_{A B A_{1} \ldots A_{p-1}} \ell_{\sigma^{A} \ldots \sigma^{A}{ }_{p-1} \mathbf{t}_{i}} \\
{\left[\ell_{\mathbf{t}_{A}}, \ell_{\sigma^{B_{1} \ldots \sigma_{p-1}} \mathbf{t}_{i}}\right] } & =\left[C^{k}{ }_{A i} \delta_{C_{1} \ldots C_{p-1}}^{B_{1} \ldots B_{p-1}}-(p-1) C_{A C_{1}}^{\left[B_{1}\right.} \delta_{C_{2} \ldots C_{p-1}}^{\left.B_{2} \ldots B_{p-1}\right]} \delta_{i}^{k}\right] \ell_{\sigma^{C_{1} \ldots \sigma}{ }^{C_{p-1}} \mathbf{t}_{k}} \\
{\left[\ell_{\sigma^{A_{1} \ldots \sigma^{A}-1} \mathbf{t}_{i}}, \ell_{\sigma^{B_{1} \ldots \sigma^{B_{p-1}}} \mathbf{t}_{j}}\right] } & =0
\end{aligned}
$$

This algebra can be considered the dual of the FDA system given in (1), (2), and extends the $G$ Lie algebra of ordinary Lie derivatives along the $\mathbf{t}_{A}$ tangent vectors of the $G$ group manifold. Notice the essential presence of the $(p-1)$-form $\sigma^{A_{1}} \ldots \sigma^{A_{p-1}}$ in front of the "tangent vectors" $\mathbf{t}_{i}$.

As a concrete example, in the case of $D=11$ supergravity the algebra (21)-(23) is given in ref. [8], and contains the supertranslation algebra with central charges discussed for ex. in refs. [19-21].

In the following we use the simplified notations

$$
T_{A} \equiv \ell_{\mathbf{t}_{A}}, \quad T_{i}^{A_{1} \ldots A_{p-1}} \equiv \ell_{\sigma^{A_{1}} \ldots \sigma^{A_{p-1}} \mathbf{t}_{i}}
$$

Nonassociativity. The dual algebra of a FDA is in general nonassociative. Indeed computing the Jacobiator of three (usual) Lie derivatives, and using the expressions (21), (22) for the commutators, yields:

$$
\begin{aligned}
J_{\mathrm{ABC}} & \equiv\left[\left[T_{A}, T_{B}\right], T_{C}\right]+\text { cyclic in } \mathrm{ABC}= \\
& =\frac{1}{(p-1) !}\left((p-1) C^{i}{ }_{E A_{2} \ldots A_{p-1}[A B} C_{C] A_{1}}^{E}-C^{j}{ }_{A_{1} A_{2} \ldots A_{p-1}[A B} C_{C] j}^{i}\right) T_{i}^{A_{1} \ldots A_{p-1}}
\end{aligned}
$$

which is nonvanishing in general ${ }^{2}$ (it is not proportional to the left-hand side of the Jacobi identities (5)). This is the main result of the present paper. Since Lie derivatives generate infinitesimal diffeomorphisms, and these are the symmetries of the theory based on the FDA, it follows that the FDA symmetries close a nonassociative algebra.

It is worthwhile to check this result in the FDA $D=11$ supergravity example, containing a 3-form $A$ in the identity representation of $G=$ superPoincaré group in 11 dimensions. Computing for example the Jacobiator (25) for two supersymmetry transformations generated by the charges $Q_{\alpha}$ and $Q_{\beta}$ and a Lorentz rotation generated by $M_{a b}$ yields

$$
J_{\alpha \beta[a b]}=\eta_{c[a}\left(C \Gamma_{b] d}\right)_{\alpha \beta} T^{c d}
$$

with $T^{c d} \equiv \ell_{V^{c} V^{d} \mathbf{t}}$. This Jacobiator does not vanish when applied to the 3 -form $A$, since $T^{c d} A=\ell_{V^{c} V^{d} \mathbf{t}} A=d\left(V^{c} V^{d}\right)$.

In the following we consider three other examples of nonassociative algebras originating from the dual formulation of FDA's.

\footnotetext{
${ }^{2}$ The nonassociativity of the dual algebra (21)-(23) was not recognized in refs. [7-9].
} 


\section{Examples.}

i) $p=2$ and $B$ in a 1-dimensional representation (with a single-valued index $i=\bullet$ ). Then the dual FDA algebra is

$$
\begin{aligned}
{\left[T_{A}, T_{B}\right] } & =C_{A B}^{C} T_{C}+C_{\mathrm{ABC}}^{\bullet} T_{\bullet}^{C} \\
{\left[T_{A}, T_{\bullet}^{B}\right] } & =-C_{A C}^{B} T_{\bullet}^{C}+C_{A \bullet}^{\bullet} T_{\bullet}^{B} \\
{\left[T_{\bullet}^{A}, T_{\bullet}^{B}\right] } & =0
\end{aligned}
$$

with a corresponding Jacobiator

$$
J_{\mathrm{ABC}}=3\left(C_{D[A B}^{\bullet} C_{C] E}^{D}-C_{E[A B}^{\bullet} C_{C] \bullet}^{\bullet}\right) T_{\bullet}^{E}
$$

Naming the generators as

$$
T_{A}=\left(x_{a}, \tilde{x}_{a}, q\right), \quad T_{\bullet}^{B}=\left(p^{b}, \tilde{p}^{a}, q^{\prime}\right)
$$

and taking as values of the structure constants

$$
C_{q a}^{b}=\delta_{a}^{b}, \quad C_{q \tilde{a}}^{\tilde{b}}=\delta_{\tilde{a}}^{\tilde{b}}, \quad C_{a b c}^{\bullet}=R_{a b c}, \quad C_{q \bullet}^{\bullet}=3
$$

(all other structure constants vanishing, the indices $\tilde{a}$ labeling the $\tilde{x}$ or $\tilde{p}$ directions, the index $q$ labeling the $q$ or the $q^{\prime}$ direction) we find that the algebra (27)-(29) contains as a subalgebra the algebra of the $R$-flux model (see [10], p. 10):

$$
\begin{aligned}
& {\left[x_{a}, x_{b}\right]=l_{s}^{3} R_{a b c} p^{c}, \quad\left[x_{a}, p^{b}\right]=i \hbar \delta_{a}^{b} q^{\prime}, \quad\left[p^{a}, p^{b}\right]=0} \\
& {\left[\tilde{x}_{a}, \tilde{x}_{b}\right]=0, \quad\left[\tilde{x}_{a}, \tilde{p}^{b}\right]=i \hbar \delta_{a}^{b} q^{\prime}, \quad\left[\tilde{p}^{a}, \tilde{p}^{b}\right]=0}
\end{aligned}
$$

where we have rescaled the generators as $x_{a} \rightarrow l_{S} x^{a}, p^{b} \rightarrow \frac{1}{l_{S}} p^{b}$ (same for $\tilde{x}_{a}$ and $\tilde{p}^{b}$ ) and $q^{\prime} \rightarrow i \hbar q^{\prime}$. The charge $q^{\prime}$ is central in this subalgebra, and therefore can play the role of the identity. The other nonvanishing commutators involving the extra charge $q$ are

$$
\left[q, x_{a}\right]=x_{a}, \quad\left[q, p^{b}\right]=2 p^{b}, \quad\left[q, \tilde{x}_{a}\right]=\tilde{x}_{a}, \quad\left[q, \tilde{p}^{b}\right]=2 \tilde{p}^{b}, \quad\left[q, q^{\prime}\right]=3 q^{\prime}
$$

Note that $q^{\prime}$ cannot be seen as identity in the whole algebra since it does not commute with $q$.

Computing the Jacobiator (30) for three generators $x_{a}, x_{b}, x_{c}$ we find it to be proportional to $\hbar l_{s}^{3} R_{a b c}$, as given in [10].

The FDA equations corresponding to the structure constants given in (32) are:

$$
\begin{aligned}
d \sigma^{a}+Q \sigma^{a} & =0 \\
d \tilde{\sigma}^{a}+Q \tilde{\sigma}^{a} & =0 \\
d Q & =0 \\
d B^{\bullet}+3 Q B^{\bullet}+C_{a b c}^{\bullet} \sigma^{a} \sigma^{b} \sigma^{c} & =0
\end{aligned}
$$


where $\sigma^{a}$ and $\tilde{\sigma}^{a}$ are 1-forms dual respectively to the coordinates $x_{a}$ and their doubles $\tilde{x}_{a}, Q$ is a one-form $\mathrm{U}(1)$ gauge field dual to the charge $q$, and $B$ is the 2 -form in a 1-dimensional $\mathrm{U}(1)$ representation (with a 1 -dimensional index $i=\bullet$ ). It is easy to verify that $d^{2}=0$ (the only nontrivial case being $d^{2}$ on $B^{\bullet}$ ), thus proving that the structure constants (32) indeed satisfy generalized Jacobi identities.

The choice of a 2-form in the FDA's allows to identify the momenta $p^{b}$ and $\tilde{p}^{b}$ as part of the "new" generators $T_{\bullet}^{A}$.

ii) $B: p$-form in the identity representation.

$$
\begin{aligned}
{\left[T_{A}, T_{B}\right] } & =C_{A B}^{C} T_{C}+\frac{1}{(p-1) !} C_{A B C_{1} \ldots C_{p-1}} T^{C_{1} \ldots C_{p-1}} \\
{\left[T_{A}, T^{B_{1} \ldots B_{p-1}}\right] } & =-(p-1) C_{A C}^{\left[B_{1} T^{\left.C B_{2} \ldots B_{p-1}\right]}\right.} \\
{\left[T^{A_{1} \ldots A_{p-1}}, T^{\left.B_{1} \ldots B_{p-1}\right]}\right.} & =0 \\
J_{\mathrm{ABC}} & =\frac{1}{(p-2) !} C_{A_{1} \ldots A_{p-1}[A B} C_{C] D}^{A_{1}} T^{D A_{2} \ldots A_{p-1}}
\end{aligned}
$$

iii) $B: 3$-form in a generic $G$ representation:

$$
\begin{aligned}
{\left[T_{A}, T_{B}\right] } & =C_{A B}^{C} T_{C}+\frac{1}{2} C^{i}{ }_{A B A_{1} A_{2}} T_{i}^{A_{1} A_{2}} \\
{\left[T_{A}, T_{i}^{B_{1} B_{2}}\right] } & =C_{A i}^{k} T_{k}^{B_{1} B_{2}}+2 C_{A C}^{\left[B_{1}\right.} T_{i}^{\left.B_{2}\right] C} \\
{\left[T_{i}^{A_{1} A_{2}}, T_{j}^{B_{1} B_{2}}\right] } & =0 \\
J_{\mathrm{ABC}} & =-\left(C^{i}{ }_{E A_{1}[A B} C_{C] A_{2}}^{E}+\frac{1}{2} C_{A_{1} A_{2}[A B}^{j} C_{C] j}^{i}\right) T_{i}^{A_{1} A_{2}}
\end{aligned}
$$

The observation in i) on the identification of an extended R-flux model algebra with a dual FDA algebra is in fact only a very preliminary step towards understanding and exploiting a possible connection between dual FDA algebras and the (double) geometry of flux backgrounds in closed string theory.

\section{Acknowledgments}

We thank Ioannis Bakas and Dieter Lüst for a discussion on the nonassociative algebras of refs. $[10,11]$.

Open Access. This article is distributed under the terms of the Creative Commons Attribution License (CC-BY 4.0), which permits any use, distribution and reproduction in any medium, provided the original author(s) and source are credited.

\section{References}

[1] R. D'Auria and P. Fré, Geometric supergravity in $D=11$ and its hidden supergroup, Nucl. Phys. B 201 (1982) 101 [Erratum ibid. B 206 (1982) 496] [INSPIRE]. 
[2] D. Sullivan, Infinitesimal computations in topology, Publ. Math. Institut Hautes Étud. Sci. 47 (1977) 269.

[3] L. Castellani, P. Fré, F. Giani, K. Pilch and P. van Nieuwenhuizen, Gauging of D $=11$ supergravity?, Annals Phys. 146 (1983) 35 [INSPIRE].

[4] P. van Nieuwenhuizen, Free graded differential algebras, in Group theoretical methods in physics, Lect. Notes Phys. 180, Springer, Berlin Germany (1983).

[5] L. Castellani, R. D'Auria and P. Fré, Supergravity and superstrings: a geometric perspective, World Scientific, Singapore (1991).

[6] L. Castellani, Group geometric methods in supergravity and superstring theories, Int. J. Mod. Phys. A 7 (1992) 1583 [inSPIRE].

[7] L. Castellani and A. Perotto, Free differential algebras: their use in field theory and dual formulation, Lett. Math. Phys. 38 (1996) 321 [hep-th/9509031] [INSPIRE].

[8] L. Castellani, Lie derivatives along antisymmetric tensors and the M-theory superalgebra, J. Math. Phys. 3 (2011) 110504 [hep-th/0508213] [INSPIRE].

[9] L. Castellani, Extended Lie derivatives and a new formulation of $D=11$ supergravity, J. Math. Phys. 3 (2011) 110505 [hep-th/0604213] [INSPIRE].

[10] I. Bakas and D. Lüst, 3-cocycles, non-associative star-products and the magnetic paradigm of $R$-flux string vacua, JHEP 01 (2014) 171 [arXiv:1309.3172] [INSPIRE].

[11] D. Lüst, T-duality and closed string non-commutative (doubled) geometry, JHEP 12 (2010) 084 [arXiv:1010.1361] [INSPIRE].

[12] D. Mylonas, P. Schupp and R.J. Szabo, Membrane $\sigma$-models and quantization of non-geometric flux backgrounds, JHEP 09 (2012) 012 [arXiv:1207.0926] [INSPIRE].

[13] O. Hohm, D. Lüst and B. Zwiebach, The spacetime of double field theory: review, remarks and outlook, Fortsch. Phys. 61 (2013) 926 [arXiv:1309.2977] [INSPIRE].

[14] R. Blumenhagen, M. Fuchs, F. Haßler, D. Lüst and R. Sun, Non-associative deformations of geometry in double field theory, JHEP 04 (2014) 141 [arXiv: 1312.0719] [INSPIRE].

[15] Y. Ne'eman and T. Regge, Gravity and supergravity as gauge theories on a group manifold, Phys. Lett. B 74 (1978) 54 [inSPIRE].

[16] Y. Ne'eman and T. Regge, Gauge theory of gravity and supergravity on a group manifold, Riv. Nuovo Cim. 1N5 (1978) 1 [inSPIRE].

[17] A. D'Adda, R. D'Auria, P. Fré and T. Regge, Geometrical formulation of supergravity theories on orthosymplectic supergroup manifolds, Riv. Nuovo Cim. 3N6 (1980) 1 [INSPIRE].

[18] R. D' Auria, P. Fré and T. Regge, Graded Lie algebra cohomology and supergravity, Riv. Nuovo Cim. 3N12 (1980) 1 [INSPIRE].

[19] E. Bergshoeff and E. Sezgin, New space-time superalgebras and their Kac-Moody extension, Phys. Lett. B 232 (1989) 96 [INSPIRE].

[20] P.K. Townsend, Four lectures on M-theory, in Proceedings of "High energy physics and cosmology", Trieste Italy (1996), pg. 385 [hep-th/9612121] [INSPIRE].

[21] P.K. Townsend, M-theory from its superalgebra, in Proceedings of "Strings, branes and dualities", Cargese France (1997), pg. 141 [hep-th/9712004] [INSPIRE]. 